\title{
A case of bilateral luxatio erecta
}

\author{
Lawrence Camarda $\cdot$ Umberto Martorana • \\ Michele D'Arienzo
}

Received: 18 July 2008/Accepted: 17 November 2008/Published online: 19 December 2008

(C) Springer-Verlag 2008

\begin{abstract}
Luxatio erecta is an uncommon form of glenohumeral dislocation. Cases of bilateral inferior shoulder dislocation (luxatio erecta) are very rare, and only ten cases have been described in literature. We describe a case of a woman with bilateral luxatio erecta of the shoulders treated with close reduction, immobilization and rehabilitation. The patient had fallen downstairs while clinging to both lateral banisters.
\end{abstract}

Keywords Shoulder - Luxatio erecta .

Bilateral inferior dislocation

\section{Introduction}

Luxatio erecta is a rare form of glenohumeral dislocation. The clinical manifestation is typical and it is characterized by abduction of the affected arm, flexion of the elbow and pronation of the forearm. Bilateral luxatio erecta represents an extremely rare condition, and just a few cases were reported in medical literature. Because of a high incidence of related complications such as neurovascular damages early recognition and treatment of this type of dislocation is required by physicians.

In this article we describe a case of a bilateral luxatio erecta that was successfully treated without long-term complications.

L. Camarda · U. Martorana · M. D'Arienzo

Dipartimento delle Discipline Chirurgiche,

Clinica Ortopedica e Traumatologica,

Università degli Studi di Palermo, Via del Vespro 125,

90127 Palermo, Italy

L. Camarda $(\square)$

Via E. Albanese 7, 90139 Palermo, Italy

e-mail: lawrencecamarda@hotmail.it

\section{Case report}

A 70-year-old female was accepted in our emergency department because of a bilateral shoulder pain that onsets after a sudden fall happened in her house. The anamnestic research showed that the patient tumbled downstairs; while clinging to both lateral banisters, she slipped down banging her face and chest to the ground.

At physical examination both shoulders were abducted and elevated approximately $90^{\circ}-100^{\circ}$ from the horizontal plane, with flexed elbows and with hands below the head. The patient was unable to adduct and lower the arms, and there was immediate pain when attempting passive movements. Both humeral heads were palpable in the lateral chest wall of the axillae. No vascular deficit was found, and the patient complained of slight paresthesias on both arms, with superficial and deep sensation deficit on both arms.

An X-ray examination was performed showing a bilateral inferior dislocation of humeral heads in relation with the glenoid fossa; no fracture was associated (Fig. 1).

Close reduction under sedation was achieved without difficulty by pushing the humeral head superiorly with an axial traction-countertraction maneuver on both shoulders. $\mathrm{X}$-ray control revealed a correct anatomic reduction of dislocations and after the reduction no vascular damage were found while paresthesias were still present on the left arm (Fig. 2). Both arms were immobilized with bilateral slings in total adduction, intrarotation with the supinated arms. The patient was discharged the day after the reduction.

The progressive mobilization of the shoulders started after 2 weeks with passive movements and pendulum Codman's exercises. After 3 weeks the patient gave up complaining paresthesias on the left arm and active movements were allowed. 


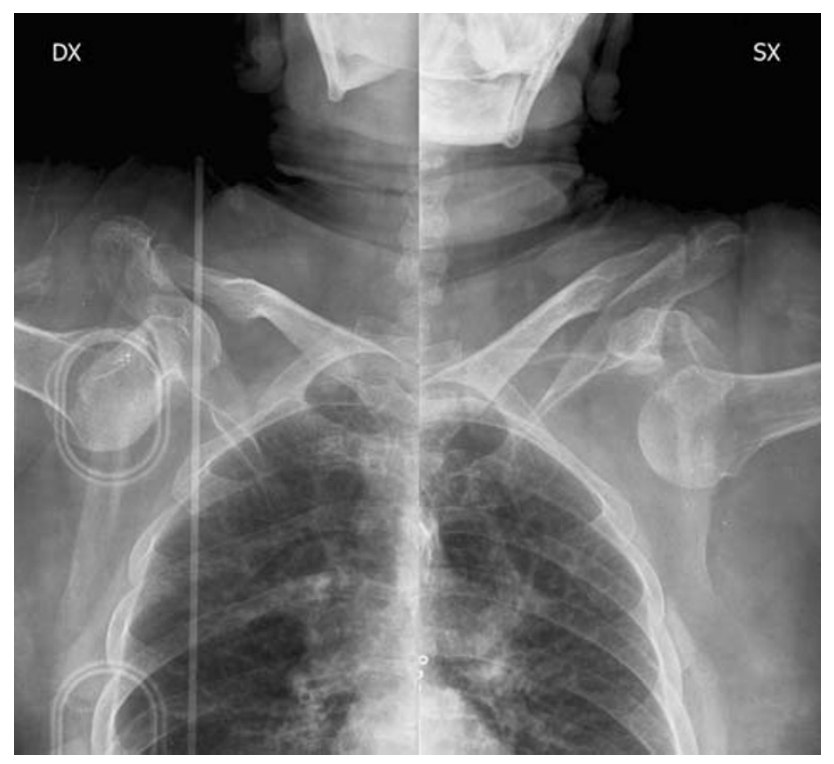

Fig. 1 Bilateral luxatio erecta



Fig. 2 Post-reduction X-ray

After 8 weeks she recovered the complete function of both shoulders and after a one-year follow-up treatment the patient showed a complete range of motion of both arms. The patient provided the informed consent for the publication of the clinical history.

\section{Discussion}

Inferior dislocations are rare $(0.5 \%$ of all shoulder dislocations) and bilateral inferior shoulder dislocations are exceptional: only 10 cases have been described so far in the literature [1].

According to the literature, luxatio erecta is due to an indirect mechanism such as a violent abduction force on abducted limb resulting in impingement of the proximal shaft of the humerus against acromion [2]. Direct axial loading from superior direction is another different mechanism, though less common. In our case the first mechanism has to be taken into consideration; in fact, while the patient was sliding in a sitting position downstairs, her hands were holding onto both banisters and the arms were abduced. When she reached the ground floor the hands were blocked around the end of the banisters and her head and body were thrown forward by gravity.

The clinical presentation of an inferior shoulder dislocation is typical: the extremities are held over the head or abduced in a fixed position with the elbow flexed. Any passive movement is possible, and the humeral head is palpable on the chest wall [3]. X-ray shows the humeral head located below the rim of the glenoid and the humeral shaft is parallel to the scapular spine [4]. In order to reduce inferior glenohumeral dislocation, it is recommended a traction counter-traction maneuver under sedation followed by immobilization in a Desault bandage for at least 2 weeks.

There is a high incidence of associate tears of the rotator cuff (12\% of patients) [5] and a SLAP lesion has been described by Schai and Hintermann [6] after a postreduction arthroscopic assessment view. Fracture of the acromion, clavicle, inferior glenoid fossa and greater tuberosity represent a complication that should need a surgical treatment [3]. Neurovascular damages are also commonly associated with inferior shoulder dislocation [5, 7]; Garcia et al. [8] described a case of bilateral luxatio erecta complicated by an axillary vein thrombosis. In general, neurologic injuries (60\% of cases) of brachial plexus, axillar, radial and ulnar nerves are more common than vascular injuries but they tend to resolve after reduction with an excellent prognosis, suggesting the neuropraxia as the main mechanism of the injury $[5,9,10]$.

Conflict of interest statement The authors declare that they have no conflict of interestrelated to the publication of this manuscript.

\section{References}

1. Laskin RS, Sedlin ED (1971) Luxatio erecta in infancy. Clin Orthop 80:126-129

2. Rockwod CA, Green DP (1984) Fractures in adult, vol 1. Lipincott, Philadelphia, pp 856-860

3. Freundlich BD (1983) Luxatio erecta. J Trauma 23(5):434-436

4. Kothari K, Berstein RM, Griffiths HJ et al (1984) Luxatio erecta. Skeletal Radiol 11:47-49

5. Mallon WJ, Bassett FH 3rd, Goldner RD (1990) Luxatio erecta: the inferior glenohumeral dislocation. J Orthop Trauma 4(1):1924

6. Schai P, Hintermann B (1998) Arthroscopic findings in luxatio erecta of the glenohumeral joint: case report and review of the literature. Clin J Sport Med 8(2):138-141 
7. Lev-El A, Adar R, Rubinstein Z (1981) Axillary artery injury in erect dislocation of the shoulder. J Trauma 21(4):323-325

8. Garcia R, Ponsky T, Brody F, Long J (2006) Bilateral luxatio erecta complicated by venous thrombosis. J Trauma 60(5):11321134
9. Davids JR, Talbott RD (1990) Luxatio erecta humeri a case report. Clin Orthop Relat Res 252:144-149

10. Wang KC, Hsu KY, Shih CH (1992) Brachial plexus injury with erect dislocation of the shoulder. Orthop Rev 21(11):1345-1347 